\title{
ASPECTOS MODERADORES DEL EMPRENDIMIENTO EN PERSONAS CON DISCAPACIDAD. UN DIAGNÓSTICO A TRAVÉS DE LA TÉCNICA DE GRUPO NOMINAL
}

\author{
Autores: Ángel Olaz Capitán \\ olazcapi@um.es \\ Universidad de Murcia \\ Pilar Ortiz García \\ portizg@um.es \\ Universidad de Murcia \\ Salvador Manzanera-Román \\ smanzanera@um.es \\ Universidad de Murcia
}

\section{Resumen}

El objetivo de este trabajo es identificar las dimensiones y los aspectos que favorecen o limitan la actividad emprendedora de las personas con discapacidad desde una perspectiva metodológica cualitativa. El análisis se llevó a cabo a través de la Técnica de Grupo Nominal. Tras el análisis de resultados, este estudio 
propone, a través del trabajo realizado con personas con discapacidad, un conjunto de intervenciones que permitirán reducir barreras y promover el emprendimiento en un colectivo sujeto a una gran vulnerabilidad.

Palabras clave: emprendimiento; diagnóstico; discapacidad; estrategias; Técnica de Grupo Nominal.

\title{
Moderating aspects of entrepreneurship in people with disabilities. A diag- nosis through the Nominal Group Technique
}

\begin{abstract}
The goal of this work is to identify the dimensions and aspects that favor and / or limit the entrepreneurial activity of people with disabilities from a qualitative methodological perspective. The analysis was carried out through the Nominal Group Technique. After the analysis of results, this study proposes, through work done with people with disabilities, a set of interventions that will reduce barriers and promote entrepreneurship in a group subject to great vulnerability.
\end{abstract}

Key words: entrepreneurship; diagnosis; disability; strategies; Nominal Group Technique.

Fecha de recepción: 26/01/2019

Fecha de aceptación: 11/04/2019

\section{INTRODUCCIÓN}

Este trabajo pretende realizar una aproximación al fenómeno del emprendimiento en aquellas personas con discapacidad orgánica, física y sensorial.

En concreto, el estudio identifica las dimensiones y aspectos que propician y/o limitan la actividad emprendedora de este colectivo. Para ello, se ha utilizado la Técnica de Grupo Nominal (en adelante, T.G.N.)․․

Dada la imprecisión con la que en ocasiones se utilizan los conceptos relacionados con el objeto de estudio, se comenzará por aclarar una serie de términos

\footnotetext{
${ }^{1}$ Este estudio se desarrolla al amparo del Proyecto Discapacidad y Emprendimiento. Análisis Competencial (CSO2016 - 75818 - R) convocatoria para el año 2016 correspondiente al Programa Estatal de Fomento de la Investigación Científica y Técnica de Excelencia, Subprograma Estatal de Generación de Conocimiento, en el marco del Plan Estatal de Investigación Científica y Técnica y de Innovación 2013. 2016 financiado por el Ministerio de Economía y Competitividad del Gobierno de España.
} 
relacionados con el concepto de discapacidad que, a menudo, pueden ser confundidos con otros aspectos tales como: enfermedad, deficiencia y/o minusvalía. Tras resolver estas cuestiones terminológicas este trabajo aborda el emprendimiento en las personas con discapacidad y las singularidades que en él concurren. A continuación, se realizará una justificación metodológica, enfatizando en el papel conferido en este estudio a la T.G.N. empleada en varios colectivos consultados (Técnicos de la Administración, Directivos y Técnicos de Asociaciones de personas con discapacidad), aunque el trabajo se centre en el emprendimiento en personas con discapacidad. Este trabajo finaliza considerando las principales conclusiones, así como un conjunto de recomendaciones que revertirían y pondrían en valor el emprendimiento de las personas con discapacidad en clave competencial.

\section{ALGUNAS CUESTIONES TERMINOLÓGICAS PREVIAS}

Según la Clasificación Internacional de Deficiencias, Discapacidades y Minusvalías (CIDDM) deben distinguirse varios y distintos aspectos (OMS, 1997) aunque estrechamente relacionados:

- La enfermedad entendida en el sentido más genérico de su expresión que comprende cualquier tipo de enfermedad, trastorno o accidente. La enfermedad está recogida por la Organización Mundial de la Salud (OMS) en su Clasificación Internacional de Enfermedades y Problemas de Salud Relacionados (CIE).

- La deficiencia que es la representación o exteriorización directa de las consecuencias de la enfermedad, manifestándose tanto en los órganos del cuerpo como en sus funciones (también se incluyen las psicológicas).

- La discapacidad hace referencia al modo en que se objetiva la deficiencia en la persona, con la consiguiente repercusión directa en su capacidad para la realización de actividades en los términos considerados normales para cualquier individuo de sus características por razón de edad, género, etc. Es, por tanto, una restricción o ausencia de lo considerado como normal.

- La minusvalía se refiere a la "socialización" de la problemática causada en una persona por las consecuencias derivadas de una enfermedad, manifestada a través de la deficiencia y/o la discapacidad, y que afecta al desempeño del papel social que le es propio. Este concepto lleva implícito un componente socio-cultural que ayuda en su formulación y comprensión.

Esta diferenciación resulta clave para delimitar distintos conceptos que, aun estando íntimamente relacionados, presentan matices diferentes. 
Según el Real Decreto 1971/1999, de 23 de diciembre, por el que se determina el procedimiento para el reconocimiento, declaración y calificación del grado de minusvalía, las discapacidades pueden clasificarse en tres grandes grupos:

- Las discapacidades físicas ${ }^{2}$ que se encuentran vinculadas al cuerpo, miembros y órganos. Se originan en los sistemas músculo-esquelético, nervioso, respiratorio, cardiovascular, hematopoyético, digestivo, aparato urogenital, endrocrino, piel, anejos y neoplasias.

- Las discapacidades sensoriales ${ }^{3}$ asociadas al aparato visual, oído, garganta y todas aquellas estructuras relacionadas con el lenguaje.

- Las discapacidades psíquicas relacionadas con el retraso y/o enfermedad mental.

En el estudio realizado y dado las particulares características que concurren en el emprendimiento, esto es, cierto grado de iniciativa, autonomía y continuidad, las personas con discapacidad psíquica no han sido contempladas en esta fase del proyecto de investigación al requerir de un acompañamiento por parte de otra persona que vulnerara los principios rectores antes formulados.

\section{EL EMPRENDIMIENTO EN LAS PERSONAS CON DISCAPACIDAD}

A pesar de los avances sociales de las últimas décadas, la integración de las personas con discapacidad sigue siendo una cuestión pendiente. Mejorar la empleabilidad de este colectivo es un reto social de primera magnitud. Entre los objetivos enunciados por programas tales como Europa 2020 de la Comisión Europea, figura la consecución de sociedades inclusivas. En este sentido, una de las primeras medidas es conocer los distintos problemas a los que se enfrentan colectivos en riesgo

${ }^{2}$ Las discapacidades físicas, son de tipo motórico y/o visceral. Fundamentalmente son las siguientes: Sistema músculo esquelético (deficiencias articulares, amputaciones...); Sistema nervioso (epilepsia, tetraplejia, paraplejia...); Aparato respiratorio (asma, trasplante de pulmón, fibrosis quística...); Sistema cardiovascular (cardiopatías, arritmias...); Sistema hematopoyético y sistema inmunitario (anemias, Inmunodeficiencias no secundarias a infección por VIH...); Aparato digestivo (enfermedad del hígado, incontinencia...); Aparato genitourinario (deficiencias del riñón, incontinencia urinaria...); Sistema endocrino (hipertiroidismo, diabetes...); Piel y anejos (soriasis...) y Neoplasias (tumores).

${ }^{3}$ Las discapacidades sensoriales, entre las que se mencionan las auditivas y las visuales. La Discapacidad Sensorial auditiva recoge la carencia, disminución o deficiencia de la capacidad auditiva parcial o total. Dependiendo del momento en que esta se haya producido, el itinerario educativo y/o rehabilitador, pueden encontrarse personas que realizan la lectura labio-facial y que puedan comunicarse oralmente, hasta otras cuya comunicación se hace a través del lenguaje de signos. La Discapacidad Sensorial visual se refiere a la carencia o afectación del canal visual de adquisición de la información. Se observan diferentes grados de limitación la ceguera y la baja visión.

icade. Revista cuatrimestral de las Facultades de Derecho y Ciencias Económicas y Empresariales, $n^{\circ} 106$ enero-abril 2019, ISSN: 2341-0841 
de exclusión social para diseñar políticas que la combatan en cualquiera de sus formas. Las personas con discapacidad forman uno de los colectivos más expuestos a situaciones de vulnerabilidad.

Dada la amplitud del concepto y la variada casuística que recoge, resulta oportuno aclarar lo que se entenderá por discapacidad, concepto establecido por la Clasificación Internacional del Funcionamiento, de la Discapacidad y de la Salud, que define la discapacidad como un término genérico que engloba deficiencias, limitaciones de actividad y restricciones para la participación de la persona en actividades consideradas normales para cualquier individuo de sus características. La discapacidad denota los aspectos negativos de la interacción entre personas con un problema de salud (como parálisis cerebral, síndrome de Down o depresión) y factores personales y ambientales (como actitudes negativas, transporte y edificios públicos inaccesibles, y falta de apoyo social). Así se pone de manifiesto en el Informe Mundial de Discapacidad de la Organización Mundial de la Salud (OMS, 2001).

Según este informe, el colectivo de personas con discapacidad supone más de mil millones en el mundo (un 15\% de la población mundial según las estimaciones de 2010 recogidas en la Encuesta Mundial de Salud). En España, la información que se recoge en la base Estatal del Instituto de Mayores y Servicios Sociales (IMSERSO), es la relativa al histórico de las personas que han solicitado el reconocimiento de la situación de discapacidad, así como el resultado de las valoraciones efectuadas por los equipos de evaluación de las distintas comunidades autónomas, incluidas las ciudades autónomas de Ceuta y Melilla (con información actualizada a 31 de diciembre de 2015). Del resultado de este proceso de valoración se obtiene un total de 2.998 .639 personas con discapacidad, es decir, con un grado de discapacidad reconocido igual o mayor al 33\%, de acuerdo con lo que establece el artículo 4.2. del Real Decreto Legislativo 1/2013, de 29 de noviembre, por el que se aprueba el Texto Refundido de la Ley General de derechos de las Personas con Discapacidad y de su Inclusión Social.

Atendiendo a las tipologías, las discapacidades se distribuyen de la siguiente forma: osteoarticular $(28,05 \%)$, enfermedad crónica $(18,01 \%)$; mental $(16,06 \%$; neuro-muscular (10,13\%); intelectual (8,96\%); visual (7,00\%); auditiva $(5,79 \%)$; mixta $(2,67 \%)$; expresiva $(0,50 \%)$ y un porcentaje residual correspondiente a las categorías "otras" y "no consta".

Estas cifras se mantienen al alza debido a varios factores: por una parte, la evolución demográfica en la que se observa un proceso cada vez más intenso del envejecimiento; por otro, los modos de vida, que han producido un incremento global de enfermedades crónicas (tales como problemas cardiovasculares, trastornos mentales, diabetes, etc.), sin olvidar otros derivados de factores ambientales y también sociales. 
Estos datos colocan a este colectivo en una situación de vulnerabilidad importante, dada la mayor probabilidad de caer en situaciones de exclusión social. Una de las formas más importantes de inclusión es el trabajo, la consecución de un empleo y su desempeño. El trabajo constituye una de las formas más eficaces de proporcionar cohesión social, a la vez que independencia, seguridad económica y sentido de pertenencia social para individuo.

La información sobre inserción laboral de personas con discapacidad aparece recogida en diversas fuentes. En España se han realizado tres macro-encuestas por parte del Instituto Nacional de Estadística (INE) en 1999 y 2008: la Encuesta sobre Discapacidades, Deficiencias y Minusvalías (EDDM, 1986), la Encuesta sobre Discapacidades, Deficiencias y Estado de Salud (EDDS, 1999) y la última Encuesta de Discapacidad, Autonomía personal y situaciones de Dependencia (EDAD, 2008). No obstante, el contenido de estas encuestas, siendo esencial para el conocimiento del colectivo, contiene datos muy básicos sobre actividad económica y, en ningún caso sobre intención o materialización de algún tipo de actividad emprendedora. Dicha información sí aparece en la encuesta "El Empleo de las personas con discapacidad" del INE, aunque con un limitado nivel de desagregación y detalle.

Este vacío parcial se pone de manifiesto en la producción científica sobre el tema, que es tratado de forma transversal en diferentes disciplinas (Sociología, Psicología, Medicina, etc.), sin que en ellas se acabe consolidando una especialidad propiamente reconocida. La Sociología da cuenta de una reseñable producción científica desde finales de los años ochenta. En España cabe mencionar los estudios de Jiménez Lara (1992; 1998a y b; 2001; 2002; 2005; 2007); Casado (1991); Jiménez y Huete (2002a y b, 2003); Rodríguez Cabrero (2002); Rodríguez y Pérez (2004); De Lorenzo (2003); Zarco y García de la Cruz (2004); Pérez Bueno (2004); Fernández Enríquez (2005); Bascones (2006); Romañach (2002); Romañach y Palacios (2007) y los de Vázquez Ferreira (2005, 2007a y b, 2008, 2010 y 2011), entre otros.

No obstante, esta producción queda lejos de la generada sobre este tema en la tradición investigadora anglosajona. Desde dicha tradición se parte de la hipótesis que lleva a concebir la discapacidad como una construcción social que estigmatiza a las personas con algún tipo de limitación de sus capacidades físicas o psíquicas. Esta idea lleva a que las políticas activas dirigidas a la inclusión de personas con discapacidad se introduzcan en la agenda social, ya que se trata de una cuestión que afecta a todos.

La discapacidad es un fenómeno multidimensional que afecta a la persona desde una perspectiva biológica, psicológica y social. De todas ellas, la dimensión social ha sido una de las menos estudiadas (Vázquez Ferreira, 2008). Sin embargo, la determinación de los factores sociales sobre los biológicos y psicológicos es 
clara, de ahí la oportunidad de estudios que propicien actuaciones encaminadas a la normalización e integración plena de las personas con discapacidad en los entornos en los que interactúan.

El entorno laboral resulta clave para conseguir la normalización a la que se ha hecho referencia y, en particular, las experiencias emprendedoras. Conocer las posibilidades de las personas con discapacidad a partir del diagnóstico de competencias que las hacen especiales para acometer empresas de valor social, ecológico y humano, se convierte en un objetivo fundamental de cualquier sociedad que aspire a alcanzar la calificación de "inclusiva".

Algunas fuentes como el observatorio Global Entrepreneurship Monitor (GEM), constituye una de las principales bases de datos internacional sobre la actividad emprendedora. Sin embargo, no incluye un apartado específico que permita el seguimiento de dicha acción en personas con discapacidad.

De todo ello se desprende que, si bien se está avanzando en la recopilación de información y se dispone de fuentes estadísticas y estudios sobre el tema de discapacidad y emprendimiento, no resultan suficientes y, sobre todo, no son lo suficientemente específicas sobre esta cuestión.

\section{CUESTIONES METODOLÓGICAS}

La herramienta empleada en esta investigación ha sido la T.G.N. A través de este instrumento, se definieron en primer lugar las dimensiones o ámbitos de estudio del emprendimiento en personas con discapacidad a través de la realización de 4 TT.GG.NN. formadas por diferente número de personas (p) Directivos (5); Técnicos de Asociaciones (8); Técnicos de la Administración Pública (7) y Personas con Discapacidad (7).

Este trabajo se ocupa, más concretamente, de la T.G.N. ${ }^{4}$ realizada a personas con discapacidad, entendiendo que las características de este colectivo son las que mejor pueden ayudar a comprender sus limitaciones, así como el desarrollo de estrategias para su puesta en valor. La muestra estructural está compuesta por un total de 7 personas ( 4 mujeres y 3 hombres). En su composición se ha tenido en cuenta la representación de distintos tipos de discapacidad. Atendiendo a esta variable, han intervenido 4 personas con discapacidad visual y 3 con auditiva.

\footnotetext{
${ }^{4}$ Sobre el modelo original descrito para la técnica de Van de Ven y Delbecq, varios son los elementos que han sido variados en este proyecto de investigación, sin por ello querer faltar a sus objetivos principales como son: identificar y enriquecer la comprensión acerca del problema a través de un discurso susceptible de cuantificación; establecer un conjunto de hipótesis relacionadas con el significado y efectos del problema investigado y centrar la atención en las principales áreas o escenarios de investigación (Van de Ven y Delbecq, 1972, p. 338).
} 
También ha sido heterogénea desde el punto de vista de la edad, que ha oscilado entre la persona más joven, de 21 años a la más mayor, de 53. Finalmente, desde la perspectiva de la actividad emprendedora, 3 personas cuentan con ella, 1 se encuentra en su fase inicial y las otras 3, no presentan ninguna experiencia en este sentido (ver cuadro 1 ).

\section{Cuadro 1. Mapa de perfiles muestrales}

\begin{tabular}{|c|c|c|c|c|c|c|c|c|c|c|}
\hline & \multicolumn{2}{|c|}{ Sexo } & \multicolumn{3}{c|}{ Edad } & \multicolumn{2}{c|}{$\begin{array}{c}\text { Experiencia } \\
\text { emprendedora }\end{array}$} & \multicolumn{2}{c|}{$\begin{array}{c}\text { Tipo de } \\
\text { discapacidad }\end{array}$} \\
\hline T.G.N. & Hombre & Mujer & $<35$ & $35-50$ & $>50$ & SÍ & NO & $\begin{array}{c}\text { En fase } \\
\text { inicial }\end{array}$ & Visual & Auditiva \\
\hline IND.1 & & $\mathrm{X}$ & & $\mathrm{X}$ & & & $\mathrm{X}$ & & & $\mathrm{X}$ \\
\hline IND.2 & & $\mathrm{X}$ & $\mathrm{X}$ & & & & $\mathrm{X}$ & & & $\mathrm{X}$ \\
\hline IND.3 & & $\mathrm{X}$ & & & $\mathrm{X}$ & $\mathrm{X}$ & & & $\mathrm{X}$ & \\
\hline IND.4 & $\mathrm{X}$ & & $\mathrm{X}$ & & & $\mathrm{X}$ & & & $\mathrm{X}$ & \\
\hline IND.5 & & $\mathrm{X}$ & $\mathrm{X}$ & & & & & $\mathrm{X}$ & $\mathrm{X}$ & \\
\hline IND.6 & $\mathrm{X}$ & & & $\mathrm{X}$ & & $\mathrm{X}$ & & & $\mathrm{X}$ & \\
\hline IND.7 & $\mathrm{X}$ & & $\mathrm{X}$ & & & & $\mathrm{X}$ & & & $\mathrm{X}$ \\
\hline
\end{tabular}

Fuente: Elaboración propia

\section{RESULTADOS DE LA T.G.N.}

Un somero análisis de las dimensiones señaladas (ver cuadro 2) en el colectivo de personas con discapacidad indica que los aspectos donde se encuentran mayor número de limitaciones se localizan en la esfera del terreno personal y psicológico del individuo, seguido del espacio social, donde los elementos culturales son especialmente significativos. También el espacio institucional y el ámbito legal presentan elementos inhibidores del emprendimiento junto a los educativos y formativos. El terreno socio-económico y el escenario familiar, siendo importantes, no se consideran por parte de todos los colectivos como ámbitos donde pueden localizarse elementos que perjudiquen el emprendimiento. 


\section{Cuadro 2. Escenarios limitativos para personas con discapacidad}

\begin{tabular}{|l|c|}
\hline \multicolumn{1}{|c|}{ Escenarios } & T.G.N. \\
\hline Aspectos Personales-Psicológicos & $24,0 \%$ \\
\hline Aspectos Socio-Culturales & $17,0 \%$ \\
\hline Aspectos Institucionales-Legales & $25,0 \%$ \\
\hline Aspectos Educativo-Formativos & $10,0 \%$ \\
\hline Aspectos Socio-Económicos & $24,0 \%$ \\
\hline Aspectos Familiares & $0,0 \%$ \\
\hline Aspectos Físicos-Accesibilidad & $0,0 \%$ \\
\hline Total & $100,0 \%$ \\
\hline
\end{tabular}

Fuente: Elaboración propia

Profundizando un poco más en detalle en la T.G.N. realizada a personas con discapacidad sensorial y auditiva pueden distinguirse como principales rasgos:

Respecto a los factores limitadores del emprendimiento de las personas con discapacidad destacan los institucionales-legales $25 \%$, que representan el mayor peso explicativo en el conjunto de aspectos considerados. Los integrantes de la T.G.N. destacan las limitaciones que suponen para el emprendimiento cuestiones tales como la falta de vías de financiación adecuadas a las necesidades particulares de colectivos como el que nos ocupa. Junto a estos aspectos, también se denuncia la falta de oferta formativa para el emprendimiento. Otro grupo factores serían las trabas burocráticas que acompañan a los procedimientos económicos y que, para estas personas, pueden suponer una dificultad adicional. Por último, se demanda mayor sensibilidad para la eliminación de barreras físicas, así como la dotación de medios humanos y técnicos especialmente pensados para el colectivo, como pueden ser traductores.

Los aspectos personales-psicológicos junto con los socio-económicos ocupan un segundo lugar en la consideración de los participantes en esta T.G.N. con un peso del $24 \%$. En cuanto a los primeros, resultan relevantes las cuestiones derivadas de la propia discapacidad, que ralentizan aspectos como el aprendizaje. También destacan los derivados de la falta de empatía observada en la sociedad respecto a las dificultades con las que se tienen que enfrentar los personas con discpacidad en su día a día. Síntomas como la desmotivación, baja autoestima y poca confianza en sí mismos, son el resultado de la falta de comprensión percibida en el entorno. 
En cuanto a los aspectos socio-económicos (24\%), si bien no resultan específicos de las personas con discapacidad, sí tienen algunas particularidades que, en el caso de este colectivo vienen a dificultar un posible emprendimiento o su desarrollo, en caso de estar iniciado. Destacan cuestiones tales como la falta de inversión económica en apoyo técnico y humano para personas con discapacidad, falta de inversión en aspectos directamente relacionados con las cuestiones empresariales y escasos o nulos incentivos fiscales.

Seguidos de los anteriores, y con un peso explicativo en la limitación al emprendimiento del $17 \%$ se han destacado los aspectos socio-culturales. El común denominador a los límites señalados en este escenario es la falta de comprensión social sobre la discapacidad. Bajo esta premisa, los participantes relacionan cuestiones tan relevantes como las barreras en la comunicación social, la desconfianza que perciben en el resto de personas, la invisibilidad de la discapacidad y, con ella, de las dificultades que lleva aparejada cualquier actividad que afrontan. A estos aspectos se unen otros que, aun siendo transversales, como la edad, en este caso constituyen dificultades adicionales en la inserción profesional y en el emprendimiento en particular.

Por último, en cuarto lugar, los participantes en esta T.G.N. han identificado los aspectos educativo-formativos como elementos potencialmente limitadores del emprendimiento, con un peso explicativo significativamente menor que los anteriores (10\%). Entre las cuestiones mencionadas en este escenario, si bien existen elementos limitantes comunes para cualquier persona -con discapacidad o no-, como son la falta de conocimientos de marketing y otros específicos de la gestión empresarial, se enuncian otros directamente relacionados con los límites percibidos por este colectivo. Son cuestiones tales como una ausencia de formación específica para emprendedores con discapacidad u otros como la falta de recursos humanos de apoyo. Es el caso del colectivo con discapacidad auditiva usuario de la lengua de signos, que de forma reiterada denuncia la falta de intérpretes de esta forma de comunicación.

\section{CONCLUSIONES DE LA T.G.N.}

Ante la extensa relación de cuestiones que suponen un límite para el emprendimiento de las personas con discapacidad, se impone la toma de medidas que contribuyan a paliar, si no a eliminar, los obstáculos que deben superar.

Las propuestas elaboradas y recogidas tras la realización de la T.G.N. revelan un conjunto de acciones que suponen un punto de partida sobre el que comenzar a trabajar. 
Las que figuran a continuación son el resultado de la reflexión que los participantes en la T.G.N. y los propios investigadores han realizado sobre el tema:

- En lo referente a los aspectos institucionales-legales, se proponen varias soluciones ligadas a las autoridades administrativas de manera que éstas impulsen modificaciones en diversas áreas, tales como el sistema educativo, necesitado de una mayor adaptación a una formación específica que ahora solo facilita la empresa privada con costes elevados. Junto a la Administración, se proponen una serie de medidas relacionadas con la necesaria adaptación de las herramientas financieras a las necesidades especiales de las personas con discapacidad. En este sentido, la ampliación de la obra social de dichas entidades o la mejor adecuación de las líneas financieras existentes a las demandas de colectivos específicos, como es este, serían de gran ayuda para promover una actividad dotada de alto grado de autonomía como es el emprendimiento.

De forma más específica, la orientación de los recursos al aprovisionamiento de medios humanos y materiales (esencialmente tecnológicos), es una medida altamente demandada.

Desde un punto de vista competencial, las dimensiones relacionadas con la Autogestión y la Gestión de relaciones, guardan una alta correspondencia con las soluciones planteadas.

- En cuanto a los aspectos personales-psicológicos, las propuestas se orientan en dos direcciones. La primera, pone el foco de atención en la necesidad de disponer los medios necesarios para facilitar el aprendizaje, ya se trate de humanos (especialmente personal de apoyo, como intérpretes de lengua de signos para los usuarios de esta lengua) y técnicos.

La segunda dirección es la que apunta a la necesidad de disponer de recursos psicológicos que ayuden a trabajar aspectos como la autoconfianza. Una competencia que, sin duda, tiene un alto valor para cualquier acción emprendedora.

Una muestra de la relevancia del factor psico-físico es que, según los participantes en la T.G.N., todas las competencias serían necesarias a la hora de eliminar o minimizar los obstáculos para emprender.

- Las soluciones aportadas a los aspectos socio-económicos se centran en facilitar instrumentos que impulsen la actividad empresarial, como son: las líneas de crédito blando, financiación a fondo perdido y otras medidas de carácter puramente económico. No obstante, la solución no pasa por la mera disponibilidad de fondos, sino por un estudio específico sobre las necesidades financieras adaptadas a este colectivo.

Junto a las anteriores, algunas ideas son especialmente relevantes. En este sentido, se propone la creación de centros de trabajo para emprendedores con discapacidad. Desde una perspectiva competencial, este conjunto de factores estaría 
especialmente relacionado con las dimensiones de "Autogestión" y "Conciencia social".

- Las soluciones indicadas para los aspectos socio-culturales tienen que ver fundamentalmente con la sensibilización social hacia las particularidades de las personas con discapacidad. En este sentido sería necesario insistir en la importante labor que las administraciones deben realizar para visibilizar no ya a la persona con discapacidad, sino a todas sus potencialidades profesionales y personales.

La normalización de la discapacidad pasa por la inserción de estas personas en todos los ámbitos sociales y, para ello, desde la administración educativa, hasta la de ámbito económico deben estar involucradas. Es cierto que se ha avanzado en la sensibilización social hacia unas personas "diferentes", pero a la vista de las reivindicaciones realizadas, queda claro que hay mucho por hacer, de la escuela a la empresa y desde la "cuna" hasta la "tumba".

Competencialmente, en este escenario aparecen relacionadas todas las competencias. Ello muestra el esfuerzo a realizar por parte de la persona, de una forma integral, para vencer las dificultades que previamente se han enunciado.

- Por último, en el aspecto educativo-formativo las soluciones pasan por ofertar formación, pero no cualquier tipo de formación, sino aquella muy específicamente diseñada y adaptada atendiendo a las necesidades concretas del colectivo.

La accesibilidad a los recursos de forma sencilla y con asistencia personalizada es un requisito para que el esfuerzo adicional que deben hacer las personas con discapacidad a la hora de la formación, no sea un elemento disuasorio.

La eliminación de los obstáculos relacionados con la formación y su canalización en una actividad emprendedora, implica el despliegue de todas las competencias psicológicas y sociales de la persona con discapacidad.

\section{CONSIDERACIONES FINALES}

La importancia del tema analizado deriva de dos grandes dimensiones: por una parte, la necesidad de normalización en la integración laboral del colectivo de personas con discapacidad; por otra, la oportunidad de explorar todas las potencialidades de este colectivo en el desarrollo de una actividad emprendedora.

Uno y otro aspecto justifican investigaciones como la que se recoge en este artículo. Como se ha indicado en las conclusiones del trabajo, el desarrollo de las potencialidades del colectivo pasa por la desaparición de los obstáculos al emprendimiento. Estos obstáculos tienen que ver con aspectos institucionales-legales, personales-psicológicos y los económicos, fundamentalmente. Aunque se trata de dimensiones que -en principio- podrían ser comunes a cualquier colectivo, la 
aportación de soluciones pasa por llevar a cabo acciones específicas que tengan en cuenta las particularidades de las personas con discapacidad.

Sólo a partir de la acción particular sobre el colectivo, se conseguirá desarrollar el gran potencial emprendedor de las personas con discapacidad desde un punto de vista competencial. Es necesario reparar en el hecho de que a estas personas les acompaña un espíritu de superación labrado en la experiencia de vencer los obstáculos que se presentan en su vida cotidiana.

\section{BIBLIOGRAFÍA}

Bascones, L. M. \& Quezada, M. Y. (2006). Daño cerebral sobrevenido en Castilla-La Mancha: realidad sociosanitaria, desafíos de apoyo e integración comunitaria. Toledo: FISLEM.

Casado, D. (1991). Panorámica de la discapacidad. Barcelona: Intress.

Fernández, E. (2005). Representaciones de la discapacidad intelectual. Disponible en http://cisne.sim.ucm.es/search*spi/afernandez+cid/afernandez+cid/1\%2C2\%$2 \mathrm{C} 2 \% 2 \mathrm{CB} /$ frameset $\& \mathrm{FF}=$ afernandez + cid + enriquez + matilrde $\& 1 \% 2 \mathrm{C} 1 \% 2 \mathrm{C}]$.

INE (1999). Encuesta sobre Discapacidades, Deficiencias y Estado de Salud (EDDS 1999). Madrid: INE.

INE (2008). Encuesta sobre Discapacidades, Deficiencias y Minusvalías 1986 (EDDM 1986). Madrid: INE.

Jiménez, A. (1992). Tipología y Prevalencia en España de las Deficiencias. Curso de Prevención de Deficiencias. Madrid: Real Patronato de Prevención y de Atención a Personas con Minusvalía.

Jiménez, A. (1998a). El impacto de las nuevas tecnologías en el empleo de las personas con discapacidad. En M. Pimentel, La situación del empleo de las personas con discapacidad en España. Propuestas para su reactivación. Madrid: Escuela Libre Editorial.

Jiménez, A. (1998b). Jóvenes con discapacidad. Características y necesidades. Juventud y Discapacidad, 43, 13-21.

Jiménez, A. (2001). La imagen social de la discapacidad. Cuenca: Asociación Roosevelt.

Jiménez, A. (2002). Estudio sociodemográfico de la población con discapacidad en el Principado de Asturias. Oviedo: Fundación FASAD.

Jiménez A. (2005). Bases demográficas: estimación, características y perfiles de las personas en situación de dependencia. En Rodríguez, A. (dir.), Libro Blanco sobre la atención a las personas en situación de dependencia en España, (pp. 19-93). Madrid: Ministerio de Trabajo y Asuntos Sociales. 
Jiménez, A. (2007). Conceptos y tipologías de la discapacidad: documentos y normativas de clasificación más relevantes. En R. de Lorenzo \& L. Pérez (Coords.), Tratado sobre discapacidad (pp. 177-205). Navarra: Thomson Reuters-Aranzadi.

Jiménez, A. \& Huete, A. (2002a). La Discapacidad en Cifras. Madrid: IMSERSO. Jiménez, A. \& Huete, A. (2002b). La discriminación por motivos de discapacidad. Análisis de las respuestas recibidas al cuestionario sobre discriminación por motivos de discapacidad promovido por el CERMI Estatal. Madrid: CERMI.

Lorenzo, R. de (2003). El futuro de las personas con discapacidad en el mundo. Madrid: Fundación ONCE.

OMS (1997). Clasificación Internacional de Deficiencias, Discapacidades y Minusvalías. Madrid: IMSERSO.

OMS (2001). Clasificación Internacional del Funcionamiento, de la Discapacidad y de la Salud, Madrid, Ministerio de Trabajo y Asuntos Sociales. Secretaría General de Asuntos Sociales. Madrid: Instituto de Migraciones y Servicios Sociales (IMSERSO).

Pérez, L. C. (2004). El desmantelamiento de la discapacidad y otros escritos vacilantes. Barcelona: El Cobre.

Rodríguez, G. (2002). Apuntes sobre el Estado del Bienestar. Madrid: Universidad de Alcalá de Henares.

Rodríguez, G. \& Pérez, M. (2004). Pobreza y exclusión social en el Principado de Asturias. Madrid: Consejo Superior de Investigaciones Científicas.

Romañach, J. (2002). Héroes y parias: la dignidad en la discapacidad. Disponible en http://www.minusval2000.com/relaciones/vidaIndependiente/heroes_y_parias.html.

Romañach, J. \& Palacios, A. (2007). El modelo de la diversidad. La Bioética y los Derechos Humanos como herramientas para alcanzar la plena dignidad en la diversidad funcional. Valencia: Generalitat Valenciana ENIL.

Vázquez Ferreira, M. (2011). Conformaciones, performaciones y transformaciones; herramientas transductivas de la contemporaneidad. Intersticios: Revista sociológica de pensamiento crítico, 5 (2), 5-16.

Vázquez Ferreira, M. (2010). De la minus-valía a la diversidad funcional: un nuevo marco teórico-metodológico. Política y Sociedad, 47(1), 45-65.

Vázquez Ferreira, M. (2008). Una aproximación sociológica a la discapacidad desde el modelo social: apuntes caracteriológicos. Revista Española de Investigaciones Sociológicas, 124, 141-174.

Vázquez Ferreira, M. (2007a). Prácticas sociales, identidad y estratificación: tres vértices de un hecho social, la discapacidad. Intersticios. Revista Sociológica de Pensamiento Crítico, 1(2), 1-14. 
Vázquez Ferreira, M. (2007b). Un nuevo concepto para la comprensión de la acción social: la transductividad creativa de las prácticas cotidianas. Intersticios. Revista Sociológica de Pensamiento Crítico, 1(1), 1-16.

Vázquez Ferreira, M. (2005). La reflexividad social transductiva: la construcción práctico-cognitiva de lo social y la sociología. Nómadas. Revista Crítica de Ciencias Sociales y Jurídicas, 11.

Van de Ven, A. \& Delbecq, A. (1972). The nominal group as a research instrument for exploratory health studies. American Journal of Public Health, 62(3), $337-342$.

Zarco, J. \& García de la Cruz, J. J. (2004). El espejo social de la mujer con gran discapacidad. Madrid: Fundamentos. 